\title{
UNIQUENESS OF GENERATORS OF PRINCIPAL IDEALS IN RINGS OF CONTINUOUS FUNCTIONS
}

\author{
M. J. CANFELL
}

Abstract. Let $a R$ denote the principal right ideal generated in a ring $R$ by an element $a$. Kaplansky has raised the question: If $a R=b R$, are $a$ and $b$ necessarily right associates? In this note we show that for rings of continuous functions the answer is affirmative if and only if the underlying topological space is zero-dimensional. This gives an algebraic characterization of the topological concept "zero-dimensional". By extending the notion of uniqueness of generators of principal ideals we are able to give an algebraic characterization of the concept " $n$-dimensional".

In the sequel, $R$ is assumed to be commutative.

Definition. A set of principal ideals $\left\{a_{i} R\right\}, i=1, \cdots, n$, is uniquely generated if whenever $a_{i} R=b_{i} R, i=1, \cdots, n$, there exist elements $u_{i}$ of $R$ such that $a_{i}=b_{i} u_{i}, i=1, \cdots, n$, and $u_{1} R+\cdots+u_{n} R$ $=R$. The dimension of $R$-denoted by $\operatorname{dim} R$-is the least integer $n$ such that every set of $n+1$ principal ideals is uniquely generated.

In the following $X$ will denote a completely regular topological space, $C(X)$ the ring of real-valued continuous functions defined on $X$, and $C^{*}(X)$ the subring of $C(X)$ consisting of the bounded functions in $C(X)$. For $f \in C(X)$, the zero set $Z(f)$ of $f$, is defined by $Z(f)=\{x \in X: f(x)=0\}$. Clearly $f_{1} C(X)+\cdots+f_{n} C(X)=C(X)$ if and only if $Z\left(f_{1}\right) \cap \cdots \cap Z\left(f_{n}\right)=\varnothing$. For further information on zero sets the reader is referred to [2]. An important fact is that disjoint zero sets are completely separated.

We use the modification of covering dimension involving basic covers $[2$, p. 243], and the equivalent definitions given in [1]. The unit cube in $E^{n+1}$ is denoted by

$$
I^{n+1}: I^{n+1}=\left\{x \in E^{n+1}:-1 \leqq x_{i} \leqq 1, i=1, \cdots, n+1\right\} .
$$

We also write

$$
I_{+}^{n+1}=\left\{x \in E^{n+1}: 0 \leqq x_{i} \leqq 1, i=1, \cdots, n+1\right\}
$$

and

$$
S_{+}^{n}=\left\{x \in I_{+}^{n+1}: x_{i}=0 \text { or } 1 \text { for some } i\right\}
$$

Received by the editors April 1, 1970.

AMS 1969 subject classifications. Primary 1310, 5470.

Key words and phrases. Principal ideals, uniqueness of generators, dimension of a ring, rings of continuous functions, topological dimension. 
THEOREM. The following conditions are equivalent.

(i) $\operatorname{dim} X=n$.

(ii) $\operatorname{dim} C(X)=n$.

(iii) $\operatorname{dim} C^{*}(X)=n$.

Proof. $\operatorname{dim} C(X) \leqq \operatorname{dim} X$. Let $\operatorname{dim} X=n$ and suppose $f_{i} C(X)$ $=g_{i} C(X), i=1, \cdots, n+1$. There exist functions $s_{i}, t_{i}$ in $C(X)$ such that $f_{i}=g_{i} s_{i}, g_{i}=f_{i} t_{i}, i=1, \cdots, n+1$. Then $g_{i}=g_{i} s_{i} t_{i}$ so that $g_{i}\left(1-s_{i} t_{i}\right)$ $=0$. Since $Z_{i}=Z\left(s_{i}\right)$ and $Z_{i}^{\prime}=Z\left(s_{i}+1-s_{i} t_{i}\right)$ are disjoint zero sets, there exist $m_{i}$ in $C(X)$ such that $m_{i}$ is 1 on $Z_{i}, 0$ on $Z_{i}^{\prime}$ and $0 \leqq m_{i} \leqq 1$. Let $m$ be the mapping of $X$ into $I_{+}^{n+1}$ defined by $m(x)=\left(m_{1}(x), \cdots\right.$, $\left.m_{n+1}(x)\right)$. Then $m$ maps all the points in $Z_{i}$ and $Z_{i}^{\prime}$ into $S_{+}^{n}$. Since $\operatorname{dim} X \leqq n$, there exists a mapping $h=\left(h_{1}, \cdots, h_{n+1}\right)$ of $X$ into $S_{+}^{n}$ such that $h(x)=m(x)$ whenever $m(x) \in S_{+}^{n}[1$, Definition 3$]$. Let $k_{i}=s_{i}+h_{i}\left(1-s_{i} t_{i}\right), i=1, \cdots, n+1$. Then $Z\left(k_{1}\right) \cap \cdots \cap Z\left(k_{n+1}\right)=\varnothing$. To see this, note that for any $x \in X$ we have $h_{i}(x)=0$ or $h_{i}(x)=1$ for some $i$. If $h_{i}(x)=0$ then $s_{i}(x) \neq 0$ so that $k_{i}(x) \neq 0$. If $h_{i}(x)=1$, then $s_{i}(x)+1-s_{i}(x) t_{i}(x) \neq 0$ and again $k_{i}(x) \neq 0$. Hence $k_{1} C(X)+$ $\cdots+k_{n+1} C(X)=C(X)$. Finally, $g_{i} k_{i}=g_{i} s_{i}+h_{i} g_{i}\left(1-s_{i} t_{i}\right)=g_{i} s_{i}=f_{i}$, $i=1, \cdots, n+1$.

$\operatorname{dim} X \leqq \operatorname{dim} C(X)$. Let $\operatorname{dim} C(X)=n$ and let $Z_{i}, Z_{i}^{\prime}, i=1, \cdots, n+1$, be disjoint pairs of zero sets of $X$. We construct functions $f_{i}, k_{i}$ such that $f_{i}=k_{i}\left|f_{i}\right|$ and $f_{i}=1$ on $Z_{i}, f_{i}=-1$ on $Z_{i}^{\prime}, i=1, \cdots, n+1$. There exist $p_{i}$ such that $p_{i}=1$ on $Z_{i}$ and $p_{i}=-1$ on $Z_{i}^{\prime}$. There exist $k_{i}, s_{i}, t_{i}$ such that $k_{i}=1$ when $p_{i} \geqq \frac{1}{2}, k_{i}=-1$ when $p_{i} \leqq-\frac{1}{2},-1 \leqq k_{i}$ $\leqq 1$ :

$$
\begin{array}{ll}
t_{i}=1 & \text { on } \quad Z_{i}, t_{i}=0 \quad \text { when } \quad p_{i} \leqq \frac{1}{2}, 0 \leqq t_{i} \leqq 1 \\
s_{i}=-1 & \text { on } \quad Z_{i}^{\prime}, s_{i}=0 \quad \text { when } \quad p_{i} \geqq-\frac{1}{2},-1 \leqq s_{i} \leqq 0 .
\end{array}
$$

Let $f_{i}=s_{i}+t_{i}$. Then $f_{i}=k_{i}\left|f_{i}\right|,\left|f_{i}\right|=k_{i} f_{i}$, so that $f_{i} C(X)=\left|f_{i}\right| C(X)$, $i=1, \cdots, n+1$. Since $\operatorname{dim} C(X) \leqq n$, there exist $h_{1}, \cdots, h_{n+1}$ such that $f_{i}=h_{i}\left|f_{i}\right|, \quad i=1, \cdots, \quad n+1$, and $h_{1} C(X)+\cdots+h_{n} C(X)$ $=C(X)$. Clearly $Z_{i}$ and $Z_{i}^{\prime}$ are separated in $X-Z\left(h_{i}\right)$ and $Z\left(h_{1}\right)$ $\cap \cdots \cap Z\left(h_{n+1}\right)=\varnothing$. Hence $\operatorname{dim} X \leqq n[1$, Definition 4].

$\operatorname{dim} X=\operatorname{dim} C^{*}(X)$. This is proved using the same methods as above, with $C(X)$ replaced by $C^{*}(X)$.

A simple consequence of this theorem is the following well-known result.

Corollary. $\operatorname{dim} X=\operatorname{dim} \beta X=\operatorname{dim} v X$, where $\beta X$ is the Stone-Cech compactification of $X$, and $v X$ is the Hewitt real-compactification of $X$.

Proof. Since $C^{*}(X)$ is isomorphic to $C(\beta X)$, we have $\operatorname{dim} X$ 
$=n \Leftrightarrow \operatorname{dim} C^{*}(X)=n \Leftrightarrow \operatorname{dim} C(\beta X)=n \Leftrightarrow \operatorname{dim} \beta X=n$. Since $C(X)$ is isomorphic to $C(v X)$, we have $\operatorname{dim} X=n \Leftrightarrow \operatorname{dim} C(X)=n \Leftrightarrow \operatorname{dim} C(v X)=n$ $\Leftrightarrow \operatorname{dim} v X=n$.

\section{REFERENCES}

1. J. R. Gard and R. D. Johnson, Four dimension equivalences, Canad. J. Math. 20 (1968), 48-50. MR 36 \#5913.

2. L. Gillman and M. Jerison, Rings of continuous functions, The University Series in Higher Math., Van Nostrand, Princeton, N. J., 1960. MR 22 \#6994.

3. I. Kaplansky, Elementary divisors and modules, Trans. Amer. Math. Soc. 66 (1949), 464-491. MR 11, 155.

University of New England, New South Wales, Australia 